Pacific Journal of Mathematics

PRIMAL CLUSTERS OF TWO-ELEMENT ALGEBRAS 


\title{
PRIMAL CLUSTERS OF TWO-ELEMENT ALGEBRAS
}

\author{
EDWARD S. O'KeEFE
}

1. Introduction. The development of a structure theory for universal algebras which subsumes the familiar structure theory of Boolean and Post algebras and p-rings (Foster, [1]-[4]) has focused attention on certain classes of functionally complete universal algebras, called primal clusters. A primal cluster is a set of primal algebras in which every finite subset is strictly independent (see definitions $\S 2$, below). Each such cluster determines a unique subdirect factorization for each algebra satisfying all the identities common to some finite subset of the cluster. In other words, every function over a direct product of strictly independent primal algebras, expressible in terms of the algebras' operations, has a decomposition and reconstruction analogous both to the Boolean theory and the Fourier transform theory. In order to broaden the domain of application of the generalized theory, we must find strictly independent sets of primal algebras.

The purpose of this paper is to present the theory of independence of primal algebras in a new dimension. Simple necessary and sufficient conditions for strict independence of primal algebras of one primitive operation, regardless of the number of elements, have been obtained [5]. We now give necessary and sufficient conditions for strict independence of certain two-element primal algebras of the same species, regardless of the number of primitive operations.

2. Basic notions, the $\phi$-conditition. The following definitions are stated for easy reference.

Let $\mathfrak{A}=\left(A, o_{1}, o_{2}, \cdots\right)$ be a universal algebra.

2.1. The species $S p=\left[n_{1}, n_{2}, \cdots\right]$ of $\mathfrak{U}$ is the sequence of ranks of the primitive operations $o_{i}$ of $\mathfrak{A}$, where $n_{i}$ is the rank of $o_{i}$.

2.2. An expression $\phi\left(\xi_{1}, \cdots, \xi_{n}\right)$ of species $S p$ is a finite set of one or more indeterminate symbols $\xi_{i}$, composed by operation-symbols of $S p$.

2.3. A strict $\mathfrak{A}$-function is an expression interpreted in algebra $\mathfrak{Y}$. The notation $\phi=\chi(\mathfrak{H})$ means that the strict function represented by $\phi$ in algebra $\mathfrak{A}$ is the same as that for $\chi$.

2.4. $\mathfrak{A}$ is a primal algebra if every transformation of $A \times A \times \cdots \times A$ into $A$ can be represented by a strict $\mathscr{Y}$-function, and $\mathrm{S} p$ is denumerable.

Received April 25, 1960 
2.5. A finite set of algebras $\left\{\mathfrak{A}_{1}, \mathfrak{A}_{2}, \cdots, \mathfrak{A}_{p}\right\}$, all of the same species $S p$, is strictly independent if each given set of strict functions $\phi_{i}$ has a single expression $\psi$ which reduces to the given function $\phi_{i}$ in the algebra $\mathfrak{A}_{i}$; i.e., $\psi=\phi_{i}\left(\mathfrak{A}_{i}\right)$.

2.6. $\quad \widetilde{\mathfrak{A}}$ is a primal cluster if $\widetilde{\mathfrak{A}}$ is a set of primal algebras and every finite subset of $\widetilde{\mathfrak{A}}$ is strictly independent. The totality of pairwise nonisomorphic primal algebras of species [ $s$ ] constitutes a primal cluster [5]. Various other categories of primal clusters are known, largely of species $[2,1]$.

The $\phi$-condition is analogous to the factorization of functions of real numbers. It is simply that any strict function may be represented by any expression operating on some set of strict functions.

2.7. The $\phi$-condition. For every strict $\mathfrak{A}$-function, $\theta(\xi, \eta, \cdots, \zeta)$, and every strict $\mathfrak{A}$-function, $\kappa\left(\xi_{1}, \cdots, \xi_{m}\right)$, provided that no variable $\xi_{i}$ occurs twice in $\kappa$, there exist strict $\mathfrak{A}$-functions, $\psi_{1}(\xi, \eta, \cdots, \zeta), \cdots$, $\psi_{m}(\xi, \eta, \cdots, \zeta)$, such that

$$
\kappa\left(\psi_{1}(\xi, \eta, \cdots, \zeta), \cdots, \psi_{m}(\xi, \eta, \cdots, \zeta)\right)=\theta(\xi, \eta, \cdots, \zeta) .
$$

Formerly primal algebras were defined to be finite. However, this property is now derived from the denumerability of $S p$.

THEOREM 2.8. Every primal algebra is finite.

Proof. Let $\mathfrak{A}=\left\langle A, o_{1}, \cdots, o_{n}, \cdots\right\rangle$ be a primal algebra. The twovalued functions on any infinite set have a larger cardinal number than the set of expressions made of a denumerable set of operations. Therefore, the fact that the functions on $A \times A$ to $A$ are represented by expressions in the operations of $A$ means that $A$ is not infinite.

From [5], we require the following basic results.

THEOREM 2.9. In any primal algebra in which the primitive operations are onto transformations, the $\phi$-condition holds.

Theorem 2.10. Let $\mathfrak{A}=(A, o, \cdots)$ and $\mathfrak{A}=(B, o, \cdots)$ be two nonisomorphic primal algebras of the same species, Sp. Then there exists a set of unary expressions $\left\{\phi_{i}\right\}=\left\{\phi_{1}, \cdots, \phi_{p}\right\}$ of species $S p$ such that

$$
\phi_{1}=\phi_{2}=\cdots=\phi_{p}(\mathfrak{U})
$$

and such that every unary $\mathfrak{B}$-function is equivalent modulo $\mathfrak{B}$ to one of the $\phi_{1}, \cdots \phi_{p}$. 
THEOREM 2.11. Let $\left\{\mathfrak{A}_{1}, \cdots, \mathfrak{A}_{n}\right\}$ be a set of universal algebras of species Sp, in which every pair of algebras is strictly independent. If the $\phi$-condition holds in each algebra, the set is strictly independent.

3. The two-element independence theorem. Our main result is

THEOREM 3.1. Every set of primal algebras is a primal cluster if:

(i) every algebra in the set has exactly two elements,

(ii) no two algebras are isomorphic,

(iii) no primitive operation is constant,

(iv) all algebras in the set are in the same species, $S p=\left[n_{1}, \cdots\right.$, $\left.n_{m}\right]$.

The proof of Theorem 3.1 is preceded by three lemmas.

LEMMA 3.2. Let $\mathfrak{B}=\left(\left\{\beta_{1}, \beta_{2}\right\}, o, \cdots\right)$ be a two-element primal algebra with no constant primitive operations. Every expression $\phi\left(\xi_{1}\right.$, $\left.\cdots, \xi_{n}\right)$, in which no variable occurs twice may be changed, modulo algebra $\mathfrak{B}$, to any given function $\chi(\xi)$ by replacing some variable by a properly chosen strict $\mathfrak{B}$-function $\psi(\xi)$, and all others by constant strict B-functions.

Proof. If the expression $\phi\left(\xi_{1}, \cdots, \xi_{n}\right)$ has but one operation-symbol $o_{i}$, then, since no operation-symbol represents a constant, there are constants $\delta_{i}$ and $\gamma_{i}$ such that

$$
\begin{aligned}
& o_{i}\left(\delta_{1}, \cdots, \delta_{n_{i}}\right)=\chi\left(\beta_{1}\right) \\
& o_{i}\left(\gamma_{1}, \cdots, \gamma_{n_{i}}\right)=\chi\left(\beta_{2}\right) .
\end{aligned}
$$

We alter $\delta_{1}$ to $\gamma_{1}, \delta_{2}$ to $\gamma_{2}$, etc. until the function changes value. Some $j$ th argument must give the change from $\chi\left(\beta_{1}\right)$ to $\chi\left(\beta_{2}\right)$. We choose the expression $\psi(\xi)$ so that

$$
\begin{aligned}
& \psi\left(\beta_{1}\right)=\delta_{j} \\
& \psi\left(\beta_{2}\right)=\gamma_{j} \\
& o_{i}\left(\gamma_{1}, \cdots, \gamma_{j-1}, \psi\left(\beta_{k}\right), \delta_{j+1}, \cdots, \delta_{n i}\right)=\chi\left(\beta_{k}\right), \quad(k=1,2) .
\end{aligned}
$$

Since there are but two elements in the algebra $\mathfrak{B}, \chi$ is now completely represented

$$
o_{i}\left(\gamma_{1}, \cdots, \gamma_{j-1}, \psi(\xi), \delta_{j+1}, \cdots, \delta_{n_{i}}\right)=\chi(\xi) .
$$

On the other hand, let $\phi\left(\xi_{1}, \cdots, \xi_{n}\right)$ be composed of $m$ operation-symbols. Assume that the theorem holds for all expressions with fewer than $m$ operation-symbols. $\phi$ is a set of expressions $\phi_{1}, \cdots, \phi_{n_{\ell}}$ composed by 
primitive operations $o_{j}: \phi=o_{j}\left(\phi_{1}, \cdots, \phi_{n_{i}}\right) . \quad \phi_{1}, \cdots, \phi_{n_{i}}$ have fewer than $m$ operation-symbols, so by assumption,

$$
\left\{\begin{array}{l}
\phi_{k}=\gamma_{k}, \text { for } k=1, \cdots, j-1 \\
\phi_{j}=\psi(\xi) \\
\phi_{j+1}=\delta_{j+1}, \cdots, \phi_{n_{i}}=\delta_{n_{\iota}},
\end{array}\right.
$$

where all variables but one have been replaced by constants. But, obviously, in $\phi_{k}, k \neq j$, the last variable may also be replaced by a constant, since a constant result is desired and is given by either value of the variable. This leaves only one variable in $\phi_{j}$; but with these replacements

$$
\phi\left(\phi_{1}, \cdots, \phi_{n_{i}}\right)=\chi(\xi)
$$

and the proof is complete.

Lemma 3.3. If in two primal algebras $\mathfrak{A}$ and $\mathfrak{B}, \mathfrak{A}$ satisfies the $\phi$-condition, then, for every $\beta \in B$ and every $a(\xi)$, there is an expression

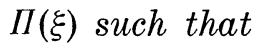

$$
\Pi(\xi)=\left\{\begin{array}{c}
a(\xi)(\mathfrak{U}) \\
\beta(\mathfrak{B}) .
\end{array}\right.
$$

Proof. Modulo $\mathfrak{B}$, there must exist expressions for constants in $B$. Therefore, letting $\kappa_{\beta}(\xi)=\beta(\mathfrak{B})$, replace each occurrence of $\xi$ in $\kappa$ by a variable from the set $\xi_{1}, \cdots, \xi_{p}$, so that in $\kappa_{\beta}\left(\xi_{1}, \cdots, \xi_{p}\right)$, no variable occurs more than once. Applying the $\phi$-condition to $\kappa_{\beta}\left(\xi_{1}, \cdots, \xi_{p}\right)$ with respect to $A$, there $\psi_{1}, \cdots, \psi_{p}$ such that

$$
\kappa_{\beta}\left(\psi_{1}, \cdots, \psi_{p}\right)=a(\xi)(\mathfrak{U}) .
$$

By Theorem 2.10, there exists a set of expressions $\left\{\phi_{\imath}\right\}$ with

$$
\phi_{i}=\psi_{i}(\mathfrak{U}) \text { and } \phi_{1}=\phi_{2}=\cdots=\phi_{p}(\mathfrak{B}) \text {. }
$$

Then

$$
\kappa_{\beta}\left(\phi_{1}, \cdots, \phi_{p}\right)=a(\xi)(\mathfrak{U}),
$$

by (3.7), but

$$
\kappa_{\beta}\left(\phi_{1}, \cdots, \phi_{p}\right)=\kappa_{\beta}\left(\phi_{1}(\xi)\right)(\mathfrak{B})=\beta(\mathfrak{B}) .
$$

Lemma 3.4. Let $\mathfrak{A}=\left(A, o_{1}, \cdots, o_{m}\right)$ be a primal algebra of species $S p$ in which every primitive operation $o_{i}$ is a transformation onto $A$. Let $\mathfrak{B}$ be a two-element primal algebra of the some species $S p$, with no constant primitive operations. Then if $\mathfrak{A}$ and $\mathfrak{B}$ are not isomorphic, they are strictly independent. 
Proof. The operations of $\mathfrak{B}$ are transformations onto $B$, since they are non-constant and $B$ has only two elements. Moreover, $\mathfrak{B}$ is primal. Therefore Theorem 2.9 applies; the $\phi$-condition holds in algebra $\mathfrak{B} . \mathfrak{A}$ is also primal with the same kind of primitive operations; hence, by Theorem 2.9, the $\phi$-condition holds for $\mathfrak{A}$ too.

Since $\mathfrak{A}$ is primal, there exists an expression, $\Sigma(\xi, \zeta)$, and an element $o \in A$ such that

$$
\begin{aligned}
& \Sigma(\xi, o)=\xi, \\
& \Sigma(o, \zeta)=\zeta .
\end{aligned}
$$

Let $p$ be the number of occurrences of $\xi$ in $\Sigma$ and $q$ the number of occurrences of $\zeta$. Replace each occurrence of $\xi$ or $\zeta$ by a different variable from the set $\left(\zeta_{1}, \cdots, \xi_{p}\right)$ or $\left(\zeta_{1}, \cdots, \zeta_{q}\right)$ respectively. Let the resulting expression be denoted $\Sigma\left(\xi_{1}, \cdots, \xi_{p}, \zeta_{1}, \cdots, \zeta_{q}\right)$. By Lemma 3.2, there exist a strict $\mathfrak{B}$-function $\psi_{j}(\zeta)$ and constant $\mathfrak{B}$-functions such that

$$
\Sigma\left(\gamma_{1}, \cdots, \gamma_{j-1}, \psi_{\jmath}(\zeta), \beta_{j+1}, \cdots, \beta_{p+q}\right)=\zeta(\mathfrak{B}) .
$$

Suppose $j \leqq p$, then by Theorem 2.10 , there are $\phi_{i}(\zeta)$ such that

$$
\phi_{i}= \begin{cases}0(\mathfrak{H}) & (i=1, \cdots, p) \\ \gamma_{i}(\mathfrak{B}) & (i=1, \cdots, j-1) \\ \psi_{j}(\xi) & (\mathfrak{B}) i=j \\ \beta_{i}(\mathfrak{B}) & (i=j+1, \cdots, p)\end{cases}
$$

and by Lemma 3.3, $\phi_{i}(\xi)$ such that

$$
\phi_{i}=\left\{\begin{array}{l}
\xi(\mathfrak{H})(i=p+1, \cdots, p+q) \\
\beta_{i}(\mathfrak{B})(i=p+1, \cdots, p+q) .
\end{array}\right.
$$

Thus,

(3.15) $\quad \Sigma\left(\phi_{1}, \cdots, \phi_{p+q}\right)=\left\{\begin{array}{l}\Sigma(0, \xi)=\xi(\mathfrak{U}) \\ \Sigma\left(\gamma_{1}, \cdots, \gamma_{j-1}, \psi_{j}(\zeta), \beta_{j+1}, \cdots, \beta p_{+} q\right)=\zeta(\mathfrak{B}) .\end{array}\right.$

An exactly similar argument shows the construction if $p<j$. Therefore, it is always possible to find an expression $\chi$ such that

$$
\chi(\xi, \zeta)=\left\{\begin{array}{l}
\xi(\mathfrak{O}) \\
\zeta(\mathfrak{B}),
\end{array}\right.
$$

and the two algebras are strictly independent by Definition 2.5.

We now return to the proof of Theorem 3.1.

Proof. Each algebra is primal, and every primitive operation is an onto transformation because none is constant and each algebra has but 
two elements. Therefore, by Theorem 2.9 , the $\phi$-condition holds in each algebra. Moreover, by Lemma 3.4, each pair of algebras is independent. Therefore, by Theorem 2.11, every finite subset of $\left\{\mathfrak{R}_{1}, \cdots\right\}$ is independent, and $\left\{\mathfrak{A}_{1}, \cdots, \mathfrak{A}_{n}, \cdots\right\}$ is a primal cluster.

\section{BIBLIOGRAPHY}

1. A. L. Foster, Generalized "Boolean" theory of universal algebras, Part 1: Subdirect sums and Normal represention theorem, Math. Zeit., 58 (1953), 306-336.

2. - Generalized "Boolean" theory of universal algebras, Part II: Identities and subdirect sums of functionally complete algebras, Math. Zeit., 59 (1953), 191-199.

3. - The identities of-and unique subdirect factorization within-Classes of universal algebras, Math. Zeit., 62 (1955), 171-188.

4. - Ideals and their structure in classes of operational Algebras, Math. Zeit., 65 (1956), 70-75.

5. E. S. O'Keefe, On the independence of primal algebras, Math. Zeit., 73 (1960), 79-94.

6. E. L. Post, The two-valued iterative systems of mathematical logic, Annals of Math Studies, Princeton, 1941. 


\section{PACIFIC JOURNAL OF MATHEMATICS}

\section{EDITORS}

\author{
RaLPh S. Phillips \\ Stanford University \\ Stanford, California \\ F. H. BRowNELL \\ University of Washington \\ Seattle 5 , Washington
}

A. L. Whiteman

University of Southern California

Los Angeles 7, California

L. J. Paige

University of California

Los Angeles 24, California

\author{
E. F. BECKENBACH \\ T. M. CHERRY
}

\author{
ASSOCIATE EDITORS

$\begin{array}{lll}\text { D. DERRY } & \text { H. L. ROYDEN } & \text { E. G. STRAUS } \\ \text { M. OHTSUKA } & \text { E. SPANIER } & \text { F. WOLF }\end{array}$

\section{SUPPORTING INSTITUTIONS}

\author{
UNIVERSITY OF BRITISH COLUMBIA \\ CALIFORNIA INSTITUTE OF TECHNOLOGY \\ UNIVERSITY OF CALIFORNIA \\ MONTANA STATE UNIVERSITY \\ UNIVERSITY OF NEVADA \\ NEW MEXICO STATE UNIVERSITY \\ OREGON STATE COLLEGE \\ UNIVERSITY OF OREGON \\ OSAKA UNIVERSITY \\ UNIVERSITY OF SOUTHERN CALIFORNIA
}

\author{
STANFORD UNIVERSITY \\ UNIVERSITY OF TOKYO \\ UNIVERSITY OF UTAH \\ WASHINGTON STATE COLLEGE \\ UNIVERSITY OF WASHINGTON \\ AMERICAN MATHEMATICAL SOCIETY \\ CALIFORNIA RESEARCH CORPORATION \\ HUGHES AIRCRAFT COMPANY \\ SPACE TECHNOLOGY LABORATORIES \\ NAVAL ORDNANCE TEST STATION
}

Mathematical papers intended for publication in the Pacific Journal of Mathematics should be typewritten (double spaced), and the author should keep a complete copy. Manuscripts may be sent to any one of the four editors. All other communications to the editors should be addressed to the managing editor, L. J. Paige at the University of California, Los Angeles 24, California.

50 reprints per author of each article are furnished free of charge; additional copies may be obtained at cost in multiples of 50 .

The Pacific Journal of Mathematics is published quarterly, in March, June, September, and December. The price per volume (4 numbers) is $\$ 12.00$; single issues, $\$ 3.50$. Back numbers are available. Special price to individual faculty members of supporting institutions and to individual members of the American Mathematical Society: $\$ 4.00$ per volume; single issues, $\$ 1.25$.

Subscriptions, orders for back numbers, and changes of address should be sent to Pacific Journal of Mathematics, 103 Highland Boulevard, Berkeley 8, California.

Printed at Kokusai Bunken Insatsusha (International Academic Printing Co., Ltd.), No. 6, 2-chome, Fujimi-cho, Chiyoda-ku, Tokyo, Japan.

\section{PUBLISHED BY PACIFIC JOURNAL OF MATHEMATICS, A NON-PROFIT CORPORATION}

The Supporting Institutions listed above contribute to the cost of publication of this Journal, but they are not owners or publishers and have no responsibility for its content or policies.

Reprinted 1966 in the United States of America 


\section{Pacific Journal of Mathematics}

\section{Vol. 11, No. 4}

A. V. Balakrishnan, Prediction theory for Markoff processes . . . . . . . . . . 1171

Dallas O. Banks, Upper bounds for the eigenvalues of some vibrating systems . . . . 1183

A. Białynicki-Birula, On the field of rational functions of algebraic groups ...... 1205

Thomas Andrew Brown, Simple paths on convex polyhedra .............. 1211

L. Carlitz, Some congruences for the Bell polynomials . . . . . . . . . . . . 1215

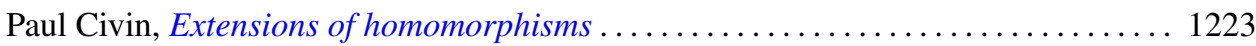

Paul Joseph Cohen and Milton Lees, Asymptotic decay of solutions of differential

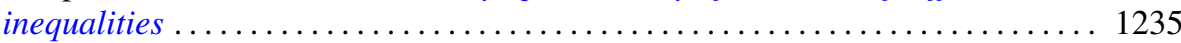

István Fáry, Self-intersection of a sphere on a complex quadric . . . . . . . . . . 1251

Walter Feit and John Griggs Thompson, Groups which have a faithful representation

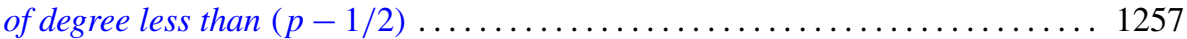

William James Firey, Mean cross-section measures of harmonic means of convex

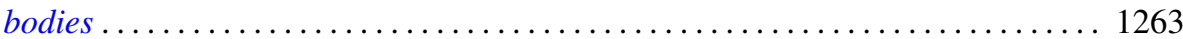

Avner Friedman, The wave equation for differential forms . . . . . . . . . . 1267

Bernard Russel Gelbaum and Jesus Gil De Lamadrid, Bases of tensor products of

Banach spaces ................................... 1281

Ronald Kay Getoor, Infinitely divisible probabilities on the hyperbolic plane . . . . 1287

Basil Gordon, Sequences in groups with distinct partial products . . . . . . . . . . . . 1309

Magnus R. Hestenes, Relative self-adjoint operators in Hilbert space . . . . . . . . . 1315

Fu Cheng Hsiang, On a theorem of Fejér ......................... 1359

John McCormick Irwin and Elbert A. Walker, On N-high subgroups of Abelian

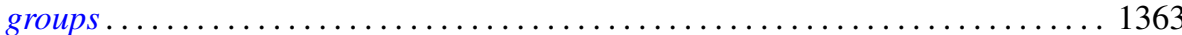

John McCormick Irwin, High subgroups of Abelian torsion groups . . . . . . . . . 1375

R. E. Johnson, Quotient rings of rings with zero singular ideal . . . . . . . . . . . 1385

David G. Kendall and John Leonard Mott, The asymptotic distribution of the time-to-escape for comets strongly bound to the solar system ...

Kurt Kreith, The spectrum of singular self-adjoint elliptic operators ....

Lionello Lombardi, The semicontinuity of the most general integral of the calculus of variations in non-parametric form ................................

Albert W. Marshall and Ingram Olkin, Game theoretic proof that Chebyshev inequalities are sharp

Wallace Smith Martindale, III, Primitive algebras with involution . . William H. Mills, Decomposition of holomorphs ..............

James Donald Monk, On the representation theory for cylindric algebras . . . . . . 1447

Shu-Teh Chen Moy, A note on generalizations of Shannon-McMillan theorem . . . . 1459

Donald Earl Myers, An imbedding space for Schwartz distributions . .

John R. Myhill, Category methods in recursion theory .........

Paul Adrian Nickel, On extremal properties for annular radial and circular slit mappings of bordered Riemann surfaces

Edward Scott O'Keefe, Primal clusters of two-element algebras . .

Nelson Onuchic, Applications of the topological method of Wazewski to certain

problems of asymptotic behavior in ordinary differential equations ...

Peter Perkins, A theorem on regular matrices................

Clinton M. Petty, Centroid surfaces .... 